\title{
REVIEW
}

\section{Analysis of structures of rubber-filler systems with combined scattering methods}

\author{
Mikihito Takenaka ${ }^{1,2}$ \\ This review presents an analysis of the hierarchical structures formed in rubber-filler systems by using combined scattering \\ methods. The combined scattering methods utilize various scattering methods and are powerful tools for the quantitative \\ characterization of hierarchical structures over a wide range of length scales, ranging from nanometers to micrometers. \\ Scattering theories for the analysis of the experimental scattering functions and their applications are described. \\ Polymer Journal (2013) 45, 10-19; doi:10.1038/pj.2012.187; published online 21 November 2012
}

Keywords: combined scattering methods; hierarchical structure; rubber-filler systems

\section{INTRODUCTION}

Rubber-filler systems have been widely used in industrial applications, such as for tires and belts so on. ${ }^{1-6}$ The use of fillers in rubber compounds reinforces the rubber material and improves the barrier properties. There are several important factors for controlling the efficiency of rubber reinforcement by fillers. The dispersion of filler particles in the rubber matrix is one of the most important factors in this process. Typically, following the use of conventional compounding processes, the fillers are not dispersed homogeneously and form hierarchical structures within the rubber matrices. For example, in rubber-carbon black (CB) systems, the $\mathrm{CB}$ primary particles are not distributed independently but coalesce into aggregates, which are indestructible units of $\mathrm{CB}$, during conventional compounding processes. ${ }^{2}$ The aggregates of $\mathrm{CB}$, which are called aggregates or agglomerates, can also lead to the formation of hierarchical structures, which consist of higher levels of ordered structure. $^{2}$ It is believed that the hierarchical structures affect the efficiency of filler reinforcement. Other rubber-filler systems also form hierarchical structures; furthermore, the characteristic lengths and morphologies of each level of ordered structure, such as agglomerates, vary with the specific combination of rubber and filler used in the system in addition to the compounding processes. To analyze the hierarchical structure, scattering techniques are one of useful techniques. In scattering experiments, we investigated the angular dependence of the scattered intensities induced by the incident beam hitting samples. Although the interpretation of the scattering intensities is complicated, nonetheless, scattering techniques are suitable for obtaining the statistical features of rubber-filler systems. However, the length scales of the hierarchical structures can extend from nanometers to micrometers, which prevents the characterization of hierarchical structures with a single scattering method. In particular, we cannot evaluate the quantitative features, such as the size and morphology of each level, from the scattering intensity obtained by a single scattering method because we cannot eliminate the possible effects of higher and lower levels of the hierarchical structure on the scattering intensity when limited to a region of small $q$ values.

In this review, I focus on the structural analysis of rubber-filler systems by using combined scattering methods. The combined scattering approaches utilize various individual scattering methods, including wide-angle scattering, small-angle scattering and ultrasmall-angle scattering with X-ray and neutron beams. In combination, the methods are powerful tools for the quantitative characterization of rubber-filler hierarchical structures over a wide range of length scales (from the nanometer to the micrometer scale). Thus, we can use these combined methods to extract useful information regarding the hierarchical structures of rubber-filler systems. Moreover, combined scattering methods enable us to investigate hierarchical structures under various sample environments, such as extension or shear deformation. We can expect to identify correlations between the mechanical properties and the respective structures during deformation processes by using combined scattering methods.

In the next section, I summarize the features of the various scattering techniques used in combined scattering methods and present the details of ultra-small-angle scattering instruments using $\mathrm{X}$-ray and neutron beams. Then, the model expressions to analyze the experimentally obtained scattering functions are described. In the subsequent section, the experimental results of the rubberfiller system scattering data are presented. Finally, I conclude the review.

${ }^{1}$ Department of Polymer Chemistry, Graduate School of Engineering, Kyoto University, Kyoto, Japan and ${ }^{2}$ Structural Materials Science Laboratory, SPring-8 Center, RIKEN Harima Institute Research, Sayo-gun, Hyogo, Japan

Correspondence: Professor M Takenaka, Department of Polymer Chemistry, Graduate School of Engineering, Kyoto University, Kyoto 615-8510, Japan.

E-mail: takenaka@alloy.polym.kyoto-u.ac.jp

Received 2 August 2012; revised 10 September 2012; accepted 10 September 2012; published online 21 November 2012 


\section{VARIOUS SCATTERING METHODS}

Figure 1 shows the wavenumbers ( $q$ regions) or length scales that can be observed with various scattering techniques and the associated hierarchical structures of the rubber-filler systems. Here, $q$ is defined by:

$$
q=\frac{4 \pi}{\lambda} \sin \theta
$$

with $\lambda$ and $\theta$ defined as, respectively, the wavelength of the incident beam and the scattering angle in the medium. The length scale $\Lambda$ is expressed by $\Lambda=2 \pi / q$. The various scattering methods are summarized in subsequent sections. Before describing the method, I must mention the $q$ region of each scattering method. The $q$ regions covered by each scattering method are described in the subsequent parts of this section. It should be noted that the region varies with the specific experimental conditions, such as the path length, the wavelength of the incident beam and the detector resolution.

\section{X-ray scattering}

Scattering methods with X-ray incident beams include wide-angle $\mathrm{X}$-ray scattering or diffraction (WAXS or XRD), small-angle X-ray scattering (SAXS), and ultra-small-angle X-ray scattering (USAXS). The scattering of X-rays is induced by fluctuations in the electron density, and the scattering power increases with atomic number. ${ }^{7}$

WAXS or XRD techniques span the range of $7<q<30 \mathrm{~nm}^{-1}$ and are widely used in the analysis of crystalline structures. For rubberfiller systems, we can observe the amorphous peaks of the rubber and the filler as well as the crystalline structure of the rubber induced by strain. ${ }^{8-10}$

The $q$ region observed with SAXS is at $0.05<q<7 \mathrm{~nm}^{-1}$. Larger $q$ regions, such as $7<q<4 \mathrm{~nm}^{-1}$, in SAXS are dominated by thermal diffusion scattering, which originates in the density fluctuations within rubbers and is related to the free volume of the amorphous region. In smaller $q$ regions in SAXS, we can observe the structures of the filler aggregates, such as the interfacial structure between the

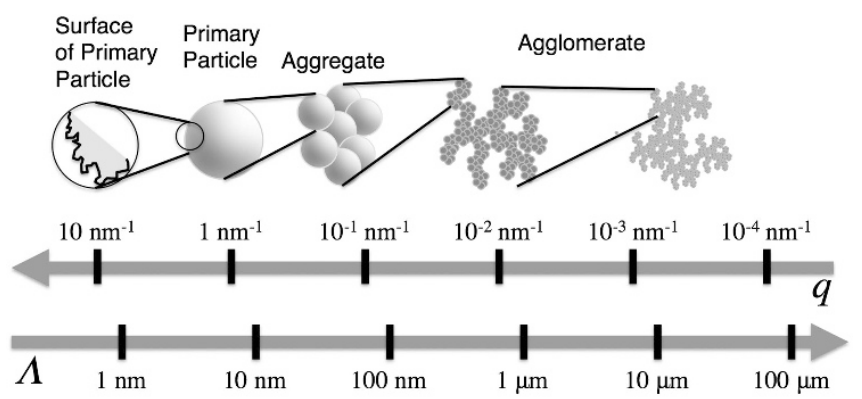

WAXS

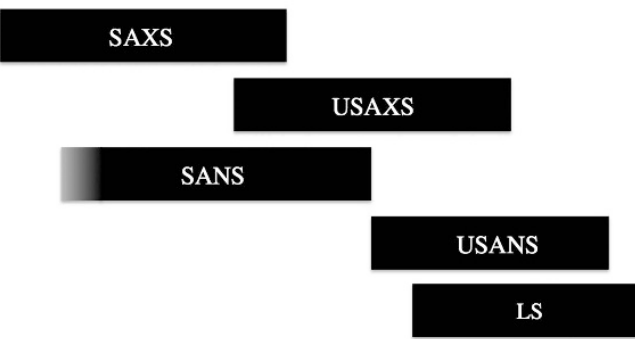

Figure $1 q$ - and length-scales covered by various scattering methods, and the hierarchical structure of rubber-filler systems. rubber and the fillers, the size and distributions of the primary filler particles, and the size of the aggregates as well as their internal structures. It should be noted that scattering from the interfacial region can not avoid the effects of thermal diffusion scattering; hence we must evaluate the thermal diffusion scattering with the method given by Ruland ${ }^{11}$ and Vonk. ${ }^{12}$ This method requires that we subtract the thermal diffusion scattering from the scattering intensity before evaluating any interfacial structures, such as the interfacial thickness. ${ }^{13,14}$ The specifics of the SAXS and USAXS analytical techniques are described later with respect to their application to aggregates.

We can observe smaller $q$ regions, such as the range of 0.001 $<q<0.05 \mathrm{~nm}^{-1}$, by using USAXS because this range is not accessible with SAXS. Thus, USAXS is useful for investigating agglomerates and the dispersion of filler aggregates in rubber.

\section{Neutron scattering}

For structural analyses of rubber-filler systems, small-angle neutron scattering (SANS) and ultra-small-angle neutron scattering (USANS) are widely used neutron scattering methods. Rubber-filler systems contain significant quantities of $\mathrm{H}$ atoms, which make it difficult to analyze the scattering at $q>1.0 \mathrm{~nm}^{-1}$ because incoherent scattering becomes dominant in this $q$ region. Unlike $\mathrm{X}$-ray scattering, neutron scattering power is independent of atomic number because neutrons interact only with atomic nuclei. ${ }^{15}$ As for typical rubber-filler systems, such as natural rubber/CB and poly(styrene-ran-butadiene)/silica, there exists a significant degree of contrast between the phases to allow for the collection of scattering function measurements. Thus, it is not necessary to synthesize deuterated polymers for neutron scattering to be able to analyze the structures of the aggregates of fillers.

SANS covers $0.01<q<1 \mathrm{~nm}^{-1}$, which is similar to the $q$ region covered by SAXS. SANS reflects the structure of the filler aggregations in addition to SAXS. We can observe the scattering intensity at $2 \times$ $10^{-4}<q<0.01 \mathrm{~nm}^{-1}$ with USANS. The $q$ region accessed by USANS reflects the agglomerate morphologies and the filler aggregate dispersions within the rubber.

Although many good reviews and books on the SAXS and SANS techniques have been published, ${ }^{7}$ there are few reviews of USAXS and USANS. Thus, I provide a brief review of USAXS and USANS in the next section.

\section{ULTRA-SMALL-ANGLE SCATTERING}

Ultra-small-angle $\mathrm{X}$-ray scattering

According to the definition of $q$ (Equation 1), we must measure the angular dependence of the scattered intensity with high angular resolution in the small-angle region because of the inherently short wavelengths of X-rays, such as $\lambda=0.154 \mathrm{~nm}$ for $\mathrm{Cu}-\mathrm{K} \alpha$. To attain such a small $q$ region (on the order of $10^{-3} \mathrm{~nm}^{-1}$ ), two techniques are available: the Bonse-Hart camera and the long path camera, which is used with synchrotron radiation X-rays.

\section{The Bonse-Hart camera for X-ray scattering}

The camera was originally invented by Bonse-Hart. ${ }^{16}$ Figure 2 presents a schematic picture of the USAXS instrumentation. ${ }^{17}$ The USAXS optics consist of the monochromator and the analyzer, both of which are fabricated from either silicon or germanium. The sample is placed between the monochromator and the analyzer. The monochromator collimates the incident X-ray source. The $q$-dependence of the scattered intensity is measured by rocking the analyzer, which allows the desired high $q$-resolution to be attained. 

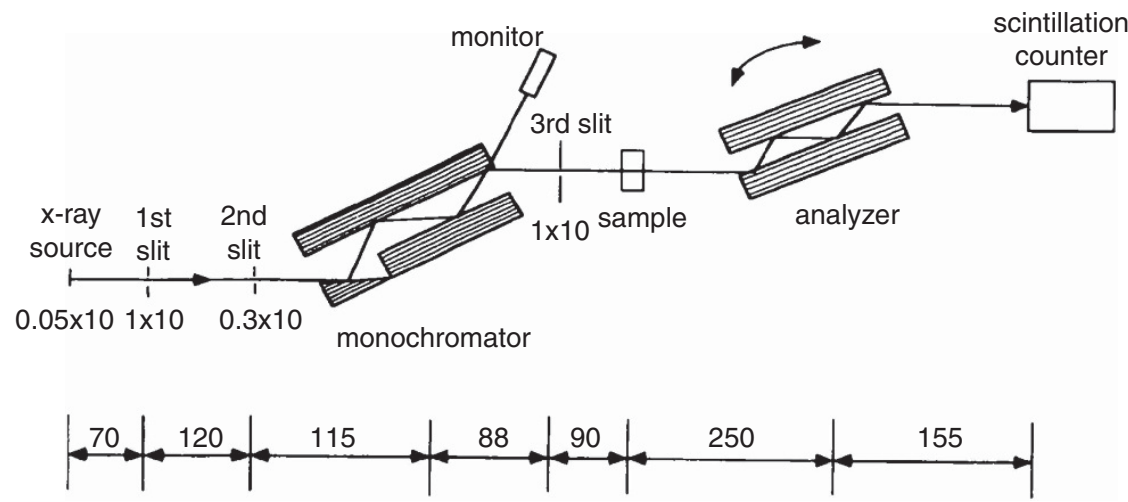

Figure 2 Side view of the optical set-up of the Bonse-Hart ultra-small-angle X-ray scattering (USAXS). Reproduced with permission from J. Appl. Crystallogr. ${ }^{17}$ Copyright (1996) International Union of Crystallography.

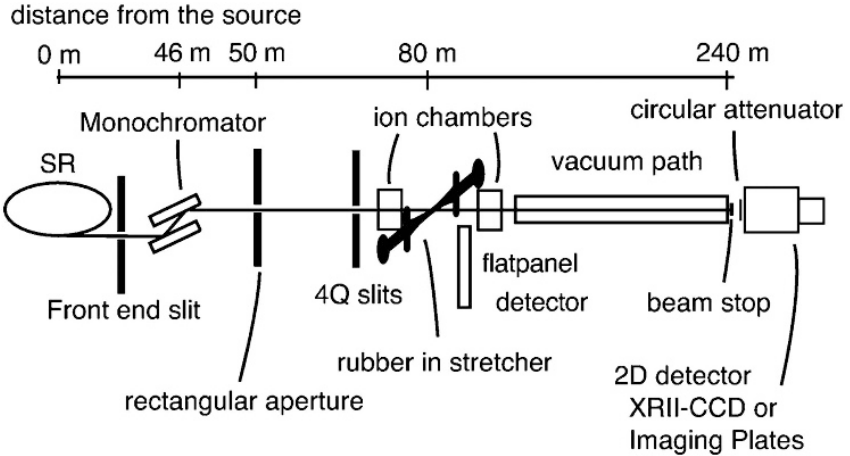

Figure 3 Schematic view of the experimental set-up of ultra-small-angle X-ray scattering (USAXS) at BL20XU. Reproduced with permission from J. Appl. Crystallogr. ${ }^{21}$ Copyright (2007) International Union of Crystallography.

We can achieve the minimum $q\left(q_{\min } \cong 1.0 \times 10^{-3} \mathrm{~nm}^{-1}\right)$ when using the Bonse-Hart camera in X-ray scattering experiments. The advantage of the Bonse-Hart camera is that conventional X-ray sources can be utilized (such as rotating anode X-ray generator) as the incident beam. However, the measured intensity is significantly affected by the smearing effects, which are caused by the incident beam profile. Thus, the use of a desmearing procedure is necessary to obtain the true scattering profile. Ultimately, based on this approach, the sample should be isotropic. Moreover, the scattered intensity is detected stepwise, which indicates that we can not follow rapid changes in structure with the Bonse-Hart camera. Recently, Bonse-Hart cameras have also been installed at synchrotron facilities. ${ }^{18,19}$ In particular, Ilavsky et al. ${ }^{20}$ succeeded in measuring two-dimensional (2D) USAXS images by using two sets of monochromators and analyzers, which were positioned both vertically and horizontally.

\section{Long path camera with synchrotron radiation X-ray}

To attain increased angular resolution, we also can use a long sampleto-detector distance. The advantage of using the long sample-todetector is that its use affords us the ability to measure 2D USAXS patterns, which can not be obtained with the Bonse-Hart camera. Figure 3 shows a schematic drawing of the USAXS setup at BL20XU, SPring-8, Hyogo, Japan. ${ }^{21,22}$ The path length at BL20XU is set to be $160 \mathrm{~m}$; with this length, the $q$-range $1.5 \times 10^{-3}$ $<q<2.5 \times 10^{-2} \mathrm{~nm}^{-1}$ can be obtained with an X-ray energy of $23 \mathrm{keV}$. For rubber-filler systems, we can collect the scattered

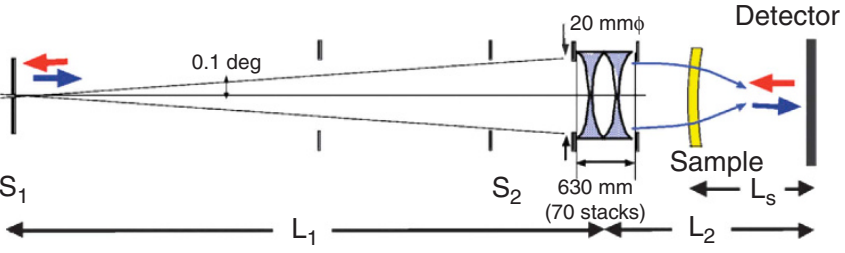

Figure 4 Schematic diagram for focusing collimation with compound lenses in which Ls $1 / 4 \quad 9.6 \mathrm{~m}, \mathrm{~L} 1 \quad 1 / 4 \quad 8.1-8.7 \mathrm{~m}$ and L2 $1 / 4 \quad 11-11.6 \mathrm{~m}$. Reproduced with permission from J. Appl. Crystallogr. ${ }^{30}$ Copyright (2001) International Union of Crystallography.

images in 2-s intervals by using a CCD detector coupled with an $\mathrm{X}$-ray image intensifier, which allows in situ measurements to be carried out under deformation. USAXS measurements also can be obtained at BL19B2, SPring-8 (http://support.spring8.or.jp/en/usaxe.html). The path length at BL19B2 is approximately $40 \mathrm{~m}$, and the corresponding $q$-range is $3.0 \times 10^{-3}<q<1.0 \times 10^{-1} \mathrm{~nm}^{-1}$ with an $\mathrm{X}$-ray energy of $18 \mathrm{keV}$. BL19B2 is a bending magnet beamline, which means that the scattered intensity of this beamline is weaker than that measured at the BL20XU undulation beamline. However, the high $q$ region at BL19B2 significantly overlaps with the typical SAXS region such that we can easily combine the USAXS patterns with SAXS patterns.

\section{Ultra-small-angle neutron scattering}

Bonse-Hart camera for neutron scattering. Bonse-Hart cameras for neutron scattering experiments are also available at neutron facilities. ${ }^{23-26}$ Silicon single crystals are used as the monochromators and the analyzers in this technique as well as in the USAXS instruments. Bonse-Hart cameras used in neutron scattering experiments can cover $2 \times 10^{-4}<q<4 \times 10^{-3} \mathrm{~nm}^{-1}$ with an incident neutron wavelength of $0.2 \mathrm{~nm}$. The value of $q_{\min } \cong 2 \times 10^{-4} \mathrm{~nm}^{-1}$ for the BonseHart camera in USANS is smaller than that of the Bonse-Hart camera in USAXS $\left(q_{\min } \cong 1.0 \times 10^{-3} \mathrm{~nm}^{-1}\right)$. This difference originates in the respective differences in the half-width at half-maximum values of the Bragg reflections $(\Delta \theta)$ in neutron scattering and $\mathrm{X}$-ray scattering; that is, $\Delta \theta$ limits the $q_{\min }$. According to the dynamical theory of 
diffraction, $\Delta \theta$ is described by: $:^{25}$

$$
\Delta \theta=\frac{b_{c} e^{-w}|F| \lambda^{2}}{\pi V_{\mathrm{c}} \sin \left(2 \theta_{\mathrm{B}}\right)}
$$

where $b_{\mathrm{c}}, e^{-\mathrm{w}}, F, \lambda, \theta_{\mathrm{B}}$ and $V_{\mathrm{c}}$ are, respectively, the coherent scattering length, the Debye-Waller factor, the structure factor of the crystal, the wavelength of the incident beam, the Bragg angle, and the volume of unit crystal. For $\mathrm{Si}, b_{\mathrm{c}}$ of neutron is significantly smaller than that of the X-ray; therefore, we can attain a smaller $q$ by using neutron.

Focusing SANS. The use of focusing collimators (biconcave $\mathrm{MgF}_{2}$ lenses) enables us to measure a lower $q$ region than that accessible by pinhole SANS. ${ }^{27}$ Figure 4 shows a schematic picture of focusing SANS at SANS-J-II, JRR-3, Tokai, Japan. ${ }^{28,29}$ A high-resolution area detector with $0.5 \mathrm{~mm}$ positional resolution was installed in addition to a $\mathrm{MgF}_{2}$ lens resulting in a value of $q_{\min } \cong 3 \times 0^{-3} \mathrm{~nm}^{-1}$ is attained; in contrast, the $q_{\text {min }}$ of pinhole SANS is $3 \times 10^{-3} \mathrm{~nm}^{-1}$. Although the $q_{\min }$ of focusing SANS is larger than that of USANS, we can obtain $2 \mathrm{D}$ scattering patterns and measure anisotropic structures, such as the scattering pattern of rubber-filler systems under extension. Focusing SANS is also available at SANS-U, JRR- $3^{30}$ and several neutron facilities. ${ }^{31-33}$

Notably, the smearing effects also affect the scattering function obtained by use of the long path camera with synchrotron radiation $\mathrm{X}$-ray and focusing SANS even though the effects are weaker than those for the Bonse-Hart camera.

\section{SCATTERING THEORY FOR RUBBER-FILLER SYSTEMS}

\section{The unified Guinier/power-law}

As described previously, rubber-filler systems form hierarchical structures. By combining the various scattering methods described in the previous section, we can obtain the scattering profiles over five decades in $q$ scale. To describe the scattering profiles of the rubberfiller systems over five decades, Beuacage proposed a unified Guinier/ power-law method. ${ }^{34,35}$ According to this method, the hierarchical structure with an arbitrary number $n$ of hierarchical levels can be described by:

$$
I(q)=\sum_{i=1}^{n}\left\{G_{\mathrm{i}} \exp \left(\frac{-q^{2} R_{\mathrm{g}, \mathrm{i}}^{2}}{3}\right)+B_{\mathrm{i}} \exp \left(\frac{-q^{2} R_{\mathrm{g}, \mathrm{i}+1}^{2}}{3}\right)\left[\frac{\left(\operatorname{erf}\left(q R_{\mathrm{g}, \mathrm{i}} / \sqrt{6}\right)\right)^{3}}{q}\right]^{P_{i}}\right\}
$$

where $G_{\mathrm{i}}, B_{\mathrm{i}}$ and $P_{\mathrm{i}}$ are, respectively, the Guinier prefactor of the $i$-th level structure with a radius of gyration $R_{\mathrm{g}, \mathrm{i}}$, a prefactor of the power-law scattering of the $i$-th level structure, and the exponent of the power-law scattering, which characterizes the shape of the $i$-th level structure. $i=1$ refers to the largest-size structural level. If the structure has a sharp interface, the scattering is proportional to $q^{-4}$ or $P_{\mathrm{i}}=4$ and $B_{\mathrm{i}}=2 \pi \Delta a^{2} S$, where $S$ is the surface area of the $i$-th level structure and $\Delta a$ is the electron density or the scattering length density of the $i$-th level structure. ${ }^{36-39}$ If the interface has finite interfacial thickness, $q^{-4}$ is modified to be $q^{-4} \exp \left(-t_{\mathrm{I}}^{2} q^{2} / 2\right)$, where $t_{\mathrm{I}}$ is the interfacial thickness. For a surface fractal,

$$
B_{\mathrm{i}}=4 \pi^{2} \Delta a^{2} R_{\mathrm{g}, \mathrm{i}}^{(6-P)} \Gamma\left(\left(P_{\mathrm{i}}-1\right) \sin \left(\pi\left(P_{\mathrm{i}}-3\right) / 2\right)\right)\left(P_{\mathrm{i}}-3\right)
$$

and $P_{\mathrm{i}}=6-d_{\mathrm{s}}$, where $d_{\mathrm{s}}$ is the surface fractal dimension. ${ }^{40}$ For a mass fractal structure, $P_{\mathrm{i}}=d_{\mathrm{f}}$, where $d_{\mathrm{f}}$ is the mass fractal dimension and $1<d_{\mathrm{f}}<3$. $B_{\mathrm{i}}$ is expressed by:

$$
B_{\mathrm{i}}=\frac{G_{\mathrm{i}} d_{\min }}{R_{\mathrm{g}, \mathrm{i}}^{d_{\mathrm{f}}}} \Gamma\left(\frac{d_{f}}{2}\right)
$$

if the $i$-th level structure or aggregation is monodisperse. ${ }^{41,42}$ In Equation (5), $d_{\min }$ is called the minimum dimension and $d_{\min }=$ $d_{\mathrm{f}}$ if the aggregation is linear. For a Gaussian chain, $B_{\mathrm{i}}$ is given by:

$$
B_{\mathrm{i}}=\frac{2 G_{\mathrm{i}}}{R_{\mathrm{g}, \mathrm{i}}^{2}} .
$$

While

$B_{\mathrm{i}}=\frac{G_{\mathrm{i}}}{R_{\mathrm{g}, \mathrm{i}}^{2}}$

for a disk. The parameter $c$ is defined by:

$$
c=\frac{d_{\min }}{d_{\mathrm{f}}}
$$

which characterizes the degree of branching and is estimated from $B_{\mathrm{i}}$, $\mathrm{d}_{\mathrm{f}}$ and $R_{\mathrm{g}, \mathrm{i}}$, which are determined by fitting the experimental scattering profiles. ${ }^{42}$

When the aggregation is polydisperse, $B_{\mathrm{i}}$ is given by:

$$
B_{\mathrm{i}}=\frac{G_{\mathrm{i}} A}{R_{\mathrm{g}, \mathrm{i}}^{d_{\mathrm{f}}}} \Gamma\left(\frac{d_{\mathrm{f}}}{2}\right)
$$

and $A$ characterizes the polydispersity of the aggregation. For the branched polydisperse aggregates, it is not possible to isolate the effects of dispersion from branching. ${ }^{42}$

\section{Percus-Yevick approximation with an upper limit}

In some rubber-filler systems, such as aggregates of silica particles, the primary particles interact with each other, and a liquid-like correlation is formed in the aggregations. In such cases, we can use the Percus-Yevick approximation to calculate the correlations and the scattering functions of the primary particles. Moreover, we must impose the upper limit, which is characterized by the aggregate size, on the scattering function.

For monodisperse systems with spherically symmetric particle shapes and interactions, the scattered intensity can be written as: ${ }^{7,36}$

$$
I(q)=n_{\mathrm{p}} \Delta a^{2} V_{\mathrm{P}}^{2} P(q) S(q)
$$

where $n_{\mathrm{P}}$ is the number density of particles, $V_{\mathrm{P}}$ is the volume of the primary particle, $P(q)$ is the form factor of the primary particle with radius $R$ and $S(q)$ is the structure factor. For dilute systems, $S(q)=1$. The form factors for typical structures have been calculated and are listed in the review written by Pederesen. ${ }^{43}$ For example, the form factor $P(q, R)$ for a sphere with radius $R$ is given by:

$$
P(q)=\frac{9[\sin (q R)-q R \cos (q R)]^{2}}{(q R)^{6}} .
$$

We can obtain the analytical form of $S\left(q, R_{\mathrm{HS}}\right)$ using the PercusYevick approximation for the hard-sphere model with an interaction radius $R_{\mathrm{HS}}:{ }^{44,45}$

$$
S\left(q, R_{\mathrm{HS}}\right)=\frac{1}{1+24 \phi G\left(R_{\mathrm{HS}} q\right) /\left(R_{\mathrm{HS}} q\right)} .
$$

Here,

$$
\begin{aligned}
G(A) & =\alpha(\sin A-A \cos A) / A^{2}+\beta\left(2 A \sin A+\left(2-A^{2}\right) \cos A-2\right) / A^{3} \\
& +\gamma\left[-A^{4} \cos A+4\left\{\left(3 A^{2}-6\right) \cos A+\left(A^{3}-6 A\right) \sin A+6\right\}\right] / A^{5}
\end{aligned}
$$


and

$$
\begin{aligned}
& \alpha=(1-2 \phi)^{2} /(1-\phi)^{4} \\
& \beta=-6 \phi(1+\phi / 2)^{2} /(1-\phi)^{2} \\
& \gamma=\alpha \phi / 2 .
\end{aligned}
$$

For the polydisperse case, we cannot write the scattered intensity as a product of the form factor and the structure factor; hence, it is necessary to calculate the following equation:

$$
\begin{aligned}
I(q)= & \Delta a^{2}\left[\int_{0}^{\infty} N(r) V(r)^{2} F(q, r)^{2} d r\right. \\
& \left.+\int_{0}^{\infty} \int_{0}^{\infty} N(r) V(r) N\left(r^{\prime}\right) V\left(r^{\prime}\right) F(q, r) F(q, r) S\left(q, r, r^{\prime}\right) d r d r^{\prime}\right]
\end{aligned}
$$

In Equation (17), $N(r)$ is the number density of a particle with radius $r, V(r)$ is the volume of a particle with radius $r, F(q, r)$ is the amplitude of the form factor with $r$ and $S\left(q, r, r^{\prime}\right)$ is the partial structure factor. Vrij, ${ }^{46}$ Blum and Stell, ${ }^{47}$ Salacuse and Stell, ${ }^{48}$ and Griffith et al. ${ }^{49}$ presented the analytical scattering functions for polydisperse systems. However, this scattering function is not suitable for analyzing the experimental data. Thus, the approximated equations are usually used to analyze the experimental data. For low-volume fractions, the decoupling approximation given by Chen et al. ${ }^{50}$ is applicable:

$$
I(q)=n_{\mathrm{P}} \Delta a^{2}\left[\left\langle V(r)^{2} F(q, r)\right\rangle+\langle V(r) F(q, r)\rangle^{2} S\left(q,\left\langle r^{3}\right\rangle^{1 / 3}\right)\right]
$$

where \langle\rangle denotes the first moment with respect to the size distribution and $S(q, r)$ is given by Equations (12)-(16). For dense systems, the local monodisperse approximation proposed by Pedersen $^{51}$ is effective:

$$
I(q)=n_{\mathrm{P}} \Delta a^{2} \int_{0}^{\infty} N(r) V(r)^{2} F(q, r)^{2} S\left(q, R_{\mathrm{HS}}(r)\right) d r
$$

where $R_{H S}(r)=r$ for particles interacting with their actual radius.

The scattering functions described above do not include the upper limit of the aggregation. Here, we shall introduce the upper limit using paracrystal theory. ${ }^{52}$ For simplicity, we consider the monodisperse systems with spherical symmetry of the particle shapes and the interactions. The structure factor $S(q)$ is expressed with the radial distribution function $g(r)$ by:

$$
S(q)=1+n_{\mathrm{P}} \int_{0}^{\infty} g(r) \frac{\sin q R}{q r} 4 \pi r^{2} d r .
$$

Substituting

$$
g(r)=1+[g(r)-1]
$$

into Equation (20) to separate the scattering of the homogeneous portion, we can then obtain: ${ }^{36,53,54}$

$$
\begin{aligned}
S(q) & =1+n_{\mathrm{P}} \int_{0}^{\infty} \frac{\sin q R}{q r} 4 \pi r^{2} d r+n_{\mathrm{P}} \int_{0}^{\infty}[g(r)-1] \frac{\sin q R}{q r} 4 \pi r^{2} d r \\
& =1+n_{P} \delta(q)+n_{\mathrm{P}} \int_{0}^{\infty}[g(r)-1] \frac{\sin q R}{q r} 4 \pi r^{2} d r
\end{aligned}
$$

where $\delta(q)$ is the delta function. We usually omit either the homogeneous part or $\delta(q)$ and define $S(q)$ as:

$$
S(q)=1+n_{\mathrm{P}} \int_{0}^{\infty}[g(r)-1] \frac{\sin q R}{q r} 4 \pi r^{2} d r
$$

because $\delta(q)=0$ at $q \neq 0$. However, we must leave the term so that we can include the upper limit. Thus, we define $S(q)$ including $\delta(q)$ as $S_{\mathrm{T}}(q)$ :

$$
S_{\mathrm{T}}(q)=1+n_{\mathrm{P}} \delta(q)+n_{\mathrm{P}} \int_{0}^{\infty}[g(r)-1] \frac{\sin q R}{q r} 4 \pi r^{2} d r=n_{\mathrm{P}} \delta(q)+S(q) .
$$

According to paracrystal theory, ${ }^{52}$ the scattering function affected by the shape of the aggregation is given by:

$$
I(q)=n_{\mathrm{P}} \Delta a^{2} V_{\mathrm{P}}^{2} P(q) S_{\mathrm{T}}(q) * G(q)
$$

where $\mathrm{G}(q)$ is the structure factor of the aggregation and * denotes the convolution product. Substituting Equation (24) into Equation (25), we obtain:

$$
\begin{aligned}
& I(q)=n_{\mathrm{P}} \Delta a^{2} V_{\mathrm{P}}^{2} P(q)\left[n_{\mathrm{P}} \delta(q)+1+n_{\mathrm{P}} \int_{0}^{\infty}\left[g(r)-1 \frac{\sin q R}{q r} 4 \pi r^{2} d r\right]{ }^{\star} G(q)\right. \\
& =n_{\mathrm{P}} \Delta a^{2} V_{\mathrm{P}}^{2} P(q)\left[n_{\mathrm{P}} \delta(q)+S(q)\right] * G(q) \\
& =n_{\mathrm{P}} \Delta a^{2} V_{\mathrm{P}}^{2} P(q)\left[n_{\mathrm{P}} G(q)+S(q) * G(q)\right] .
\end{aligned}
$$

In this equation, $G(q)$ is dominant at smaller $q$ regions, whereas

$$
I(q) \approx n_{\mathrm{P}} \Delta a^{2} V_{\mathrm{P}}^{2} P(q) S(q)
$$

at higher $q$ regions because $G(q)$ is approximated by $\delta(q)$ at higher $q$ regions. With $G(q)$, we can use the Debye-Bueche equation. ${ }^{55}$ If the correlation function of $G(q)$ and $S(q)$ are known, $S(q)^{\star} G(q)$ is obtained by taking the Fourier transform of the products of the correlation functions.

By using the Percus-Yevick approximation with the upper limit, we can calculate the scattering function of the aggregations. We can incorporate the scattering function into the unified Guinier/power-law as a scattering function for one level of the hierarchical structure.

\section{The reverse Monte Carlo method}

The reverse Monte Carlo method has been effectively used to construct a possible structure in real space from its corresponding scattering function. For rubber-filler systems, the aggregation of primary silica particles has been investigated with the reverse Monte Carlo method, and real space images were obtained for the aggregations of silica particles. ${ }^{56-60}$

Hagita et al. ${ }^{61,62}$ developed a 2D pattern reverse Monte Carlo method for the analysis of $2 \mathrm{D}$ scattering patterns. They applied the method to the investigation of structural changes in silica particle filler under uniaxial elongation. From this, they calculated 3D conformations of the spherical silica particles using a series of $2 \mathrm{D}$ patterns of structure factors obtained by Shinohara et al. ${ }^{21,63}$ during elongation. They successfully obtained the deformation of the network structure formed by the silica fillers.

However, all procedures do not consider all levels of the hierarchical structure. Thus, we must eliminate the effects of the higher or lower level structures from the scattering function to quantitatively analyze the experimental data with reverse Monte Carlo methods. 
Contrast variation SANS for the analysis of adsorption layers around fillers

The contrast variation SANS method has been developed by Endo. ${ }^{64-67}$ We applied contrast variation SANS to the investigation of the adsorbed rubber layer around the silica particles and the CB. ${ }^{68,69}$ To enhance the adsorption layer, we swell the rubber-filler systems with solvent. The swollen ratio of the absorbed layers is smaller than that of the matrix phase, and the scattering contrast between the adsorption layers and the matrix phase is caused by the difference in the swollen ratio. The swollen rubber-filler system can be treated as a three-component system, and their scattering profiles can be described as follows under an incompressible condition:

$$
\begin{aligned}
I(q)= & \left(a_{\mathrm{R}}-a_{\mathrm{S}}\right)^{2} S_{\mathrm{RR}}(q)+\left(a_{\mathrm{R}}-a_{\mathrm{S}}\right)\left(a_{\mathrm{F}}-a_{\mathrm{S}}\right) S_{\mathrm{RF}}(q) \\
& +\left(a_{\mathrm{F}}-a_{\mathrm{S}}\right)^{2} S_{\mathrm{FF}}(q) .
\end{aligned}
$$

Here, $a_{\mathrm{i}}$ is the scattering length density of the $i$-th component ( $i=R$ : rubber, $F$ : filler and $S$ : solvent). $S_{\mathrm{ij}}(q)$ is the partial scattering function defined by:

$$
S_{\mathrm{ij}}(q)=\frac{1}{V} \iint\left(\delta \phi_{i}(\vec{r}) \delta \phi_{i}\left(\vec{r}^{\prime}\right)\right) \exp \left[i \vec{q}\left(\vec{r}-\vec{r}^{\prime}\right)\right] d \vec{r} d \vec{r}^{\prime}
$$

where $V$ is the scattering volume irradiated by the incident beam and $\delta \varphi(\vec{r})$ is the fluctuation of the volume fraction of $i$ at position $(\vec{r})$. We can obtain the vector of the scattering intensities $\vec{I}=\left[I_{1}(q), I_{2}(q), \ldots I_{n}(q)\right]$ from the same samples with $n$ different scattering length densities of $S$. The partial structure factors can be obtained by

$$
\vec{S}=\mathbf{M}^{T} \cdot \vec{I}
$$

where the transposed matrix $\mathbf{M}^{T}$ satisfies $\mathbf{M}^{T} \mathbf{M}=\mathbf{E}$ by singular value decomposition, $\mathbf{M}$ is the matrix of the difference in the scattering length density, and $\vec{S}$ is the vector of the partial scattering functions. For three-component systems, $\mathbf{M}$ is expressed by:

$$
\mathrm{M}=\left(\begin{array}{cccc}
{ }^{1} \Delta a_{\mathrm{R}}^{2} & { }^{1} \Delta a_{\mathrm{R}} & { }^{1} \Delta a_{\mathrm{F}} & { }^{1} \Delta a_{\mathrm{F}}^{2} \\
{ }^{2} \Delta a_{\mathrm{R}}^{2} & { }^{2} \Delta a_{\mathrm{R}} & { }^{2} \Delta a_{\mathrm{F}} & { }^{2} \Delta a_{\mathrm{F}}^{2} \\
\vdots & \vdots & & \vdots \\
{ }^{n} \Delta a_{\mathrm{R}}^{2} & { }^{n} \Delta a_{\mathrm{R}} & { }^{n} \Delta a_{\mathrm{R}} & { }^{n} \Delta a_{\mathrm{F}}^{2}
\end{array}\right)
$$

where

$$
{ }^{n} \Delta a_{R}=a_{\mathrm{R}}-{ }^{n} a_{\mathrm{S}}
$$

and

$$
{ }^{n} \Delta a_{\mathrm{F}}=a_{\mathrm{F}}-{ }^{n} a_{\mathrm{S}} .
$$

$\vec{S}$ is given by:

$$
\vec{S}=\left(\begin{array}{c}
S_{\mathrm{RR}}(q) \\
S_{\mathrm{RF}}(q) \\
S_{\mathrm{FF}}(q)
\end{array}\right) .
$$

$S_{\mathrm{FF}}(q)$ is the scattered intensity of the aggregate filler structure, whereas $S_{\mathrm{RR}}(q)$ and $S_{\mathrm{RF}}(q)$ are affected by the adsorption layer. To analyze the adsorption layer, a three-phase model consisting of the filler aggregation, the adsorption layer and the matrix phase is proposed. The application of this method is shown later.

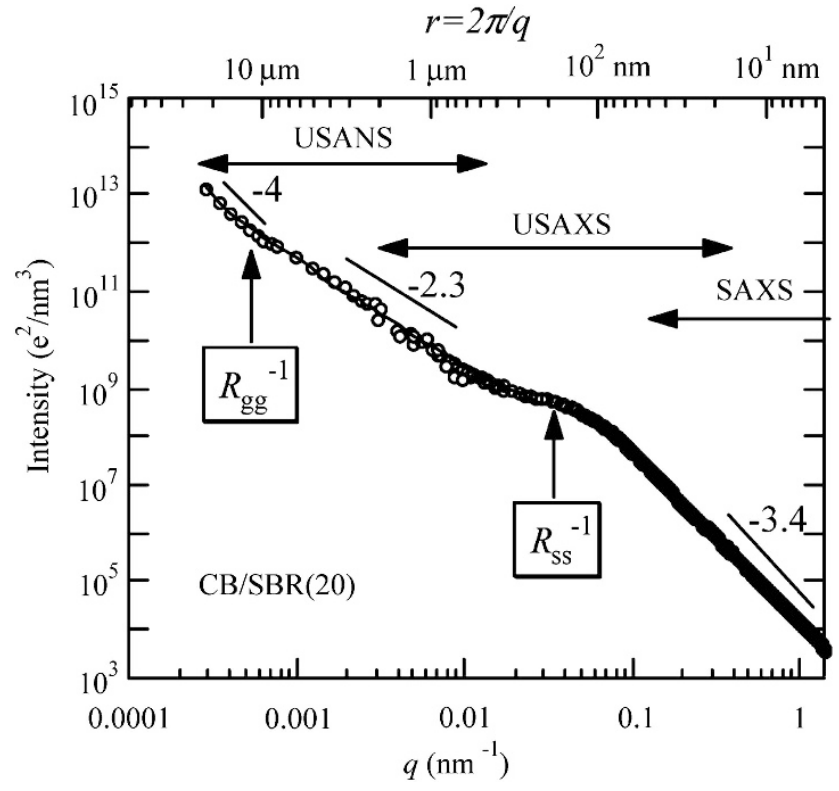

Figure $5 \mathrm{~A}$ combination of the ultra-small-angle neutron scattering (USANS), ultra-small-angle X-ray scattering (USAXS) and small-angle X-ray scattering (SAXS) profiles for carbon black (CB)/poly(styrene-randombutadiene) copolymer (SBR; open circles). $R_{\mathrm{SS}}$ and $R_{\mathrm{gg}}$ correspond to the sizes of the aggregates and the agglomerates, respectively. The solid line is the best-fitted theoretical scattering profile expressed by Equation (35). Reproduced with permission from Macromolecules. ${ }^{70}$ Copyright (2008) American Chemical Society.

\section{APPLICATIONS}

Analysis of the hierarchical structures of fillers

Koga et al. ${ }^{70,71}$ measured the scattering function of CB (SHOBLOCK N339, Showa Cabot, Chiba, Japan)/poly(styrene-random-butadiene) copolymer (SBR, weight-averaged molecular weight $M_{\mathrm{w}}=5.0 \times 10^{5}$, weight fraction of styrene content $w_{\mathrm{PS}}=0.235$ ) systems using the combined scattering method, including USANS, USAXS and SAXS, as shown in Figure 5, in which the volume fraction of CB is 0.20. They found that the scattering function of $\mathrm{CB} / \mathrm{SBR}$ can be well described by the following the unified Guinier/power-law equation:

$$
\begin{aligned}
I(q)= & A \exp \left(-q^{2} R_{\mathrm{gg}}^{2} / 3\right) q^{-4} \\
& +B \exp \left(-q^{2} R_{\mathrm{gg}}^{2} / 3\right)+C \exp \left(-q^{2} R_{\mathrm{SS}}^{2} / 3\right) \times\left[\operatorname{erf}\left(q R_{\mathrm{gg}} / \sqrt{6}\right)^{3} / q\right]^{2.3} \\
& +D \exp \left(-q^{2} R_{\mathrm{SS}}^{2} / 3\right)+E\left\{\operatorname{erf}\left(q R_{\mathrm{SS}} / \sqrt{6}\right)^{3} / q\right]^{6-D_{s}} .
\end{aligned}
$$

The fitting results and transmission electron microscope observations of the primary particles indicate that the hierarchical structure consists of the following structural levels, in increasing order of the length scale: the primary $\mathrm{CB}$ particles where the radius of the primary particles is $13 \mathrm{~nm}$ and the surface fractal structure is $D_{S}=2.6$; the aggregates comprising approximately nine fused primary $\mathrm{CB}$ particles and $R_{\mathrm{SS}}=27 \mathrm{~nm}$; the agglomerate or the mass-fractal objects formed by the aggregates with $D_{\mathrm{m}}=2.3$ and $R_{\mathrm{gg}}=2.0 \mu \mathrm{m}$; and the bulk rubber composed of a dispersion of the mass-fractal objects in the cross-linked bulk rubbers. Moreover, they found either another hierarchical structure or dispersible units between the aggregates and the agglomerates and that the morphologies of the dispersible units are an ellipsoid of revolution by investigating the detail of the 


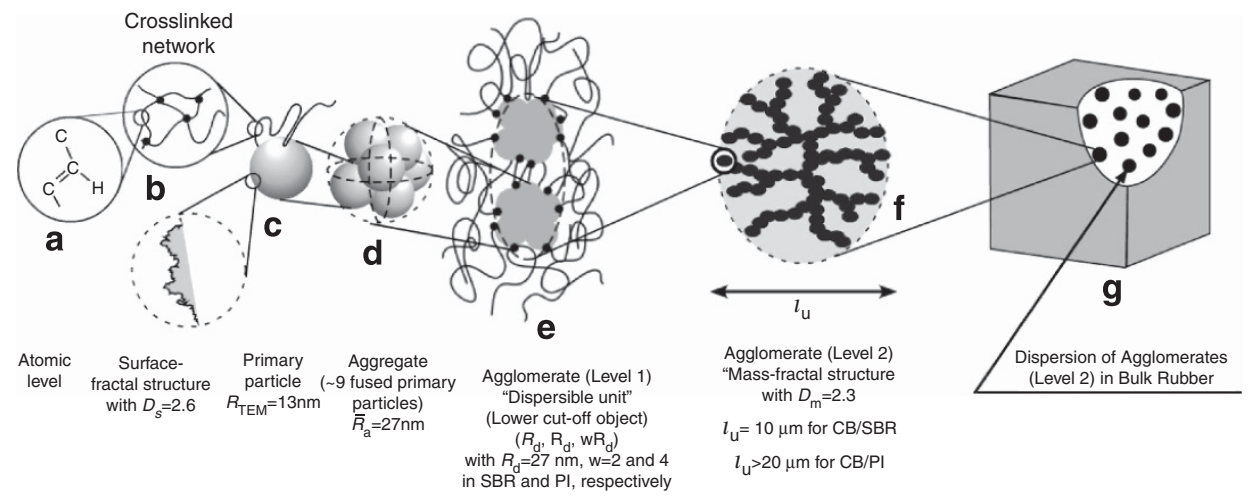

Figure 6 Schematic model of the hierarchical structure of carbon black (CB)/poly(styrene-random-butadiene) copolymer (SBR). Reproduced with permission from Macromolecules. ${ }^{70}$ Copyright (2008) American Chemical Society.

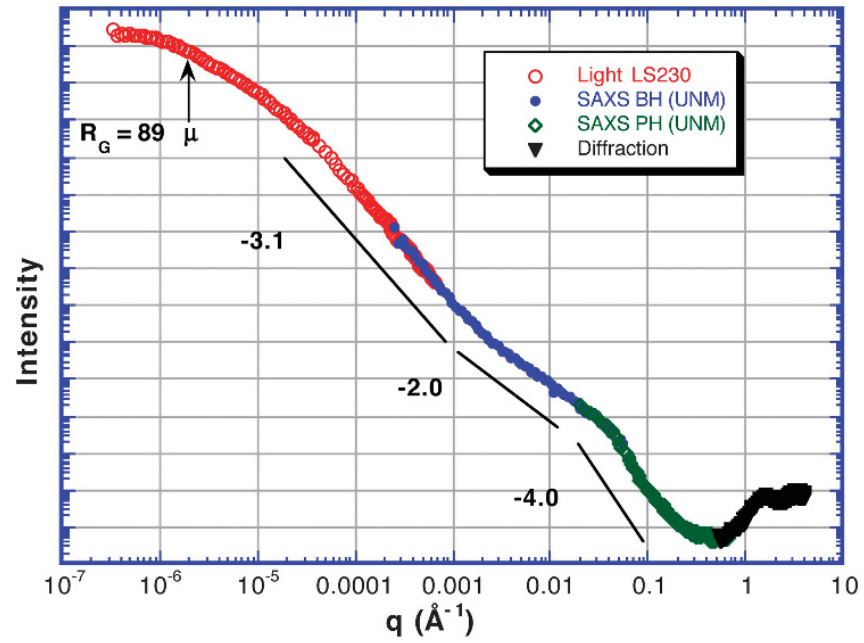

Figure 7 Scattering profile of precipitated silica. Light scattering (light), Bonse-Hart ultra-small-angle X-ray scattering (USAXS) (small-angle X-ray scattering (SAXS)-BH), SAXS (SAXS-PH) and wide-angle X-ray scattering or diffraction (WAXS; diffraction) were used. Reproduced with permission from J. Appl. Crystallogr.76 Copyright (2000) International Union of Crystallography.

scattering profiles around the Guinier region. The hierarchical structure of the SBR/CB system is summarized in Figure 6.

There are reports on applications of the unified Guinier/power-law method to silica rubber systems, precipitated silica powders and nano-composites. ${ }^{72-77}$ Silica rubber systems also form hierarchical structures consisting of agglomerates, aggregates and particles and the size of each structure can be estimated from the scattering function. Moreover, we can estimate the size distribution of silica particles. ${ }^{74}$ Schaefer et al. ${ }^{76}$ investigated the hierarchical structures of the precipitated silica prepared by the acidification of water glass using light scattering, USAXS, SAXS and WAXS, which cover the range from $3 \times 10^{-6}$ to $50 \mathrm{~nm}^{-1}$, as shown in Figure 7. By fitting four levels of the unified Guinier/power-law equation to the scattering function at $3 \times 10^{-6}<q<4 \mathrm{~nm}^{-1}$, they found that the hierarchical structure consists of agglomerate level $2\left(R_{\mathrm{g}}=115 \mu \mathrm{m}, D_{\mathrm{m}}=1.9\right.$, mass fractal $)$, agglomerate level $1\left(R_{\mathrm{g}}=0.9 \mu \mathrm{m}, D_{\mathrm{s}}=2.8\right.$, rough surface $)$, aggregates $\left(R_{\mathrm{g}}=66 \mathrm{~nm}, D_{\mathrm{m}}=1.8\right.$, mass fractal $)$ and particles $\left(R_{\mathrm{g}}=4.7 \mathrm{~nm}\right.$, $D_{\mathrm{s}}=2$ or Porod law, smooth surface). ${ }^{74}$

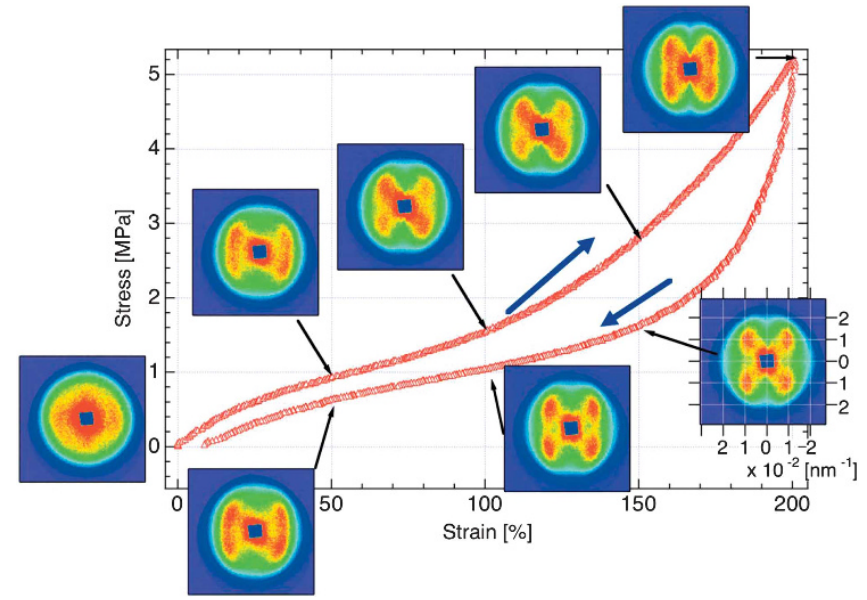

Figure 8 Stress-strain curve of poly(styrene-random-butadiene) copolymer (SBR)/silica and the corresponding two-dimensional (2D)-ultra-small-angle X-ray scattering (USAXS) images during the deformation process. Reproduced with permission from J. Appl. Crystallogr. ${ }^{21}$ Copyright (2007) International Union of Crystallography.

In situ measurement of the time-resolved 2D-USAXS of the rubberfiller systems under deformation

Shinohara et al. ${ }^{21}$ investigated the change in the structures of SBR/ silica systems with elongation using time-resolved 2D-USAXS. As shown in Figure 8, they successfully measured 2D-USAXS patterns and stress-strain curves simultaneously. They found that the distance between the silica particles increases with strain but that the distance between the silica particles connected with polymer chains remained constant. As mentioned above, Hagita et al. ${ }^{61,62}$ reconstructed the $3 \mathrm{D}$ conformations of spherical silica particles from 2D-USAXS patterns during elongation using the reverse Monte Carlo method.

\section{Analyses of the adsorption layers around fillers with contrast variation SANS}

We measured the scattering intensities of silica/SBR (systems swollen by mixtures of deuterated hexane (d-hex) and hexane (h-hex)) with various scattering length densities, as shown in Figure $9 .{ }^{68}$ By using Equations (30)-(33), we obtained partial scattering functions, as shown 


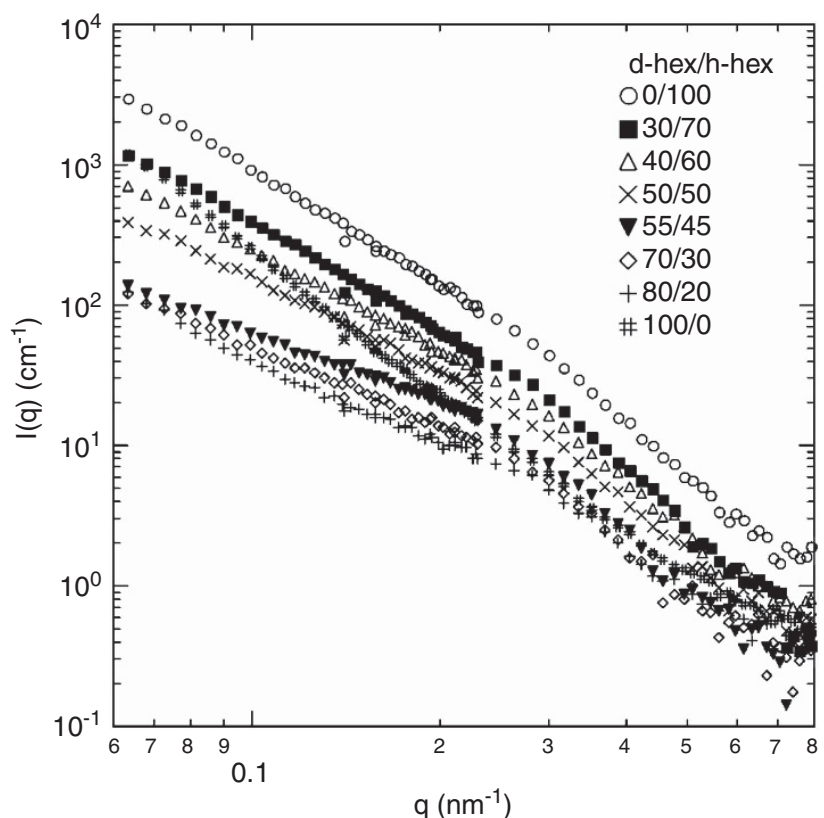

Figure 9 Scattering profiles of poly(styrene-random-butadiene) copolymer (SBR)/silica swollen by $\mathrm{d}$-hex/h-hex. Reproduced with permission from Macromolecules. ${ }^{68}$ Copyright (2009) American Chemical Society.

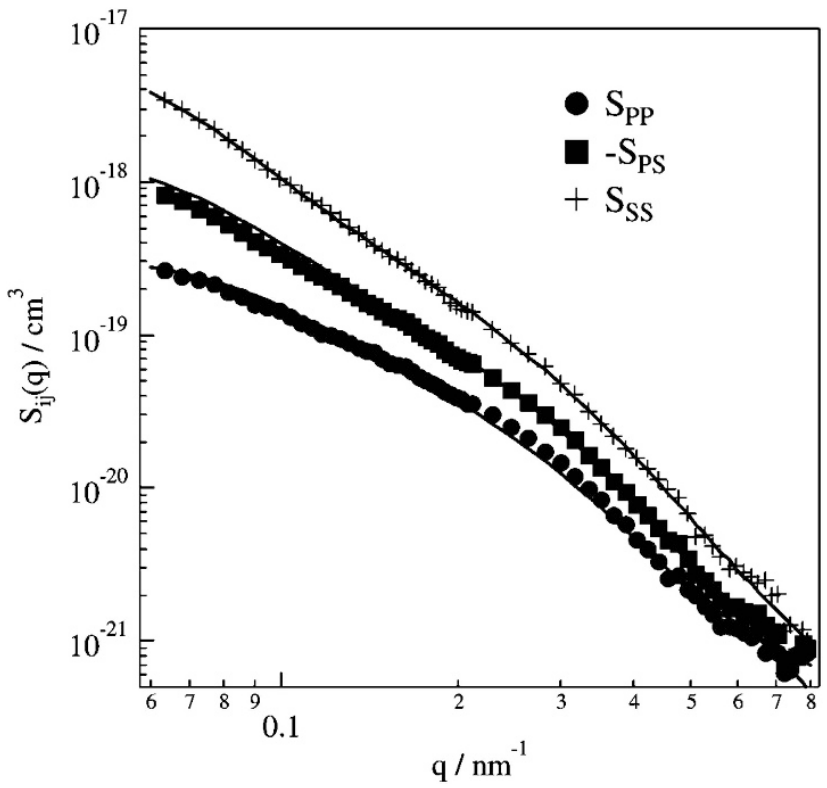

Figure 10 Partial scattering function of poly(styrene-random-butadiene) copolymer (SBR)/silica systems and their fitting results with model functions (solid lines). Reproduced with permission from Macromolecules. ${ }^{68}$ Copyright (2009) American Chemical Society.

in Figure 10: the scattering function for the polymer-polymer correlation $S_{\mathrm{PP}}(q)$, the scattering function for the silica-silica correlation $S_{S S}(q)$, and the scattering function for the polymer-silica correlation $S_{\mathrm{PS}}(q)$. The analyses of $S_{\mathrm{SS}}(q)$ explored the hierarchical structures formed by silica particles. The analyses of $S_{\mathrm{PS}}(q)$ and $S_{\mathrm{SS}}(q)$ clarified the existence of dense polymer layers around the silica aggregates. Several characteristic parameters are estimated from the analysis, such as the size of the aggregates, the thickness of the layers, the volume fractions of the polymer layers and the matrix, and the correlation length of the matrix network. We compared the volume fraction $\phi_{\mathrm{m}}$ of polymer in the matrix estimated from the SANS experiment with that from the degree of swelling and explored the agreement between them. We also estimated that the aggregation consists of 91 silica particles. In addition, we applied this method to the SBR/CB systems ${ }^{69}$ and found that the thickness of the dense polymer layers in the SBR/CB system is larger than that of the SBR/silica system.

\section{CONCLUSION}

I have described recent progress in the analysis of the hierarchical structures formed in rubber-filler systems using combined scattering methods. As the development of ultra-small-angle scattering has covered $10^{-4}<q<10^{-2} \mathrm{~nm}^{-1}$, we can quantitatively characterize hierarchical structures over a wide range of length scales, ranging from nanometers to micrometers, by combining SAXS, SANS and WAXS. It should be noted that both USAXS with a long camera path and focusing SANS are powerful tools for the analysis of change in the structures under deformation. The scattering theories for the analysis of the experimental scattering functions are summarized. The unified Guinier/power-law method can describe the scattering profiles of the rubber-filler systems with hierarchical structures. In situ 2D-USAXS measurements of the rubber-filler system under elongation can explore the deformation in the hierarchical structures. Contrast variation SANS can evaluate the interfacial properties in the rubber-filler systems.

I have not mentioned the analysis of the network structure of rubber by scattering methods, but this is an important subject in rubber-filler systems. ${ }^{78-80}$ In particular, the inhomogeneity of the network at the micron scale should be clarified.

We should explore the relationships among the mechanical properties, the structures and the compounding conditions in rubber-filler systems, which are not yet fully understood.

\section{ACKNOWLEDGEMENTS}

I would like to thank Dr Amino Naoya, Dr Yasuhiro Ishikaswa, Professor Tadanori Koga, Dr Daisuke Yamaguchi, Professor Satoshi Koizumi, Professor Shotaro Nishitsuji, Mr Toyohisa Tohyama and Mr Kiyotaka Kadomachi for their collaboration. I would like to express my gratitude to Dr Hitoshi Endo, Dr Masugu Sato, Dr Naoto Yagi, Dr Yoshio Suzuki, Dr Akihisa Takeuchi, Professor Takeji Hashimoto, Dr Hiroki Murase and Professor Masaki Takata for providing invaluable discussion and comments.

1 Boonstra, B. B. Role of particulate fillers in elastomer reinforcement-review. Polymer (Guildf) 20, 691-704 (1979)

2 Eggers, H. \& Schummer, P. Reinforcement mechanisms in carbon black and silica loaded rubber melts at low stresses. Rubber Chem. Technol. 69, 253-265 (1996).

3 Eirich, F. R. American Chemical Society \& R. D. Science and Technology of Rubber (Academic Press, 1978).

4 Heinrich, G. Advanced Rubber Composites. Vol. 238 (Springer-Verlag, Berlin, 2011).

5 Kraus, G. Reinforcement of Elastomers (Interscience Publishers, New York, 1965).

6 Nielsen, L. E. Mechanical Properties of Polymers and Composites. Vol. 1 (M. Dekker, New York, 1974)

7 Roe, R. J. Methods of X-Ray and Neutron Scattering in Polymer Science (Oxford University Press, New York, 2000)

8 Ikeda, Y., Yasuda, Y., Hijikata, K., Tosaka, M. \& Kohjiya, S. Comparative study on strain-induced crystallization behavior of peroxide cross-linked and sulfur cross-linked natural rubber. Macromolecules 41, 5876-5884 (2008).

9 Saijo, K., Zhu, Y. -P., Hashimoto, T., Wasiak, A. \& Brzostowski, N. Oriented crystallization of crosslinkedcis-1,4-polybutadiene rubber. J. Appl. Polym. Sci. 105, 137-157 (2007)

10 Toki, S., Sics, I., Hsiao, B. S., Tosaka, M., Poompradub, S. Ikeda, Y \& Kohijya, S. Probing the nature of strain-induced crystallization in polyisoprene rubber by combined thermomechanical and in situ X-ray diffraction techniques. Macromolecules 38, 7064-7073 (2005).

11 Rathje, J. \& Ruland, W. Density fluctuations in amorphous and semicrystalline polymers. Colloid Polym. Sci, 254, 358-370 (1976). 
12 Vonk, C. G. Investigation of non-ideal 2-phase polymer structures by small-angle X-ray-scattering. J. Appl. Crystallogr. 6, 81-86 (1973).

13 Hashimoto, T., Fujimura, M. \& Kawai, H. Domain-boundary structure of styreneisoprene block co-polymer films cast from solutions.5. Molecular-weight dependence of spherical microdomains. Macromolecules 13, 1660-1669 (1980).

14 Hashimoto, T., Shibayama, M. \& Kawai, H. Domain-boundary structure of styreneisoprene block co-polymer films cast from solution.4. Molecular-weight dependence of lamellar microdomains. Macromolecules 13, 1237-1247 (1980).

15 Higgins, J. S. \& Benoît, H. Polymers and Neutron Scattering (Clarendon Press; Oxford University Press, New York, 1994).

16 Bonse, U. \& Hart, M. Tailless X-ray single-crystal reflection curves obtained by multiple reflection-(Si Ge crystals-E/T). Appl. Phys. Lett. 7, 238-23 (1965).

17 Koga, T., Hart, M. \& Hashimoto, T. Development of a high-flux- and high-temperatureset-up Bonse-Hart ultra-small-angle X-ray scattering (USAXS) diffractometer. J. Appl. Crystallogr. 29, 318-324 (1996).

18 Narayanan, T., Diat, O. \& Bosecke, P. SAXS and USAXS on the high brilliance beamline at the ESRF. Nucl. Instrum. Meth. A 467, 1005-1009 (2001).

19 Sztucki, M. \& Narayanan, T. Development of an ultra-small-angle X-ray scattering instrument for probing the microstructure and the dynamics of soft matter. J. Appl. Crystallogr. 40, S459-S462 (2007).

20 llavsky, J., Jemian, P. R., Allen, A. J., Zhang, F., Levine, L. E. \& Long, G. G. Ultra-small-angle X-ray scattering at the advanced photon source. J. Appl. Crystallogr. 42, 469-479 (2009)

21 Shinohara, Y., Kishimoto, H., Inoue, K., Suzuki, Y., Takeuchi, A., Uesugi, K., Yagi, N. Muraoka, K., Mizoguchi, T. \& Amemiya, Y. Characterization of two-dimensional ultrasmall-angle X-ray scattering apparatus for application to rubber filled with spherical silica under elongation. J. Appl. Crystallogr. 40, S397-S401 (2007).

22 Yagi, N. \& Inoue, K. Ultra-small-angle X-ray diffraction and scattering experiments using medium-length beamlines at SPring-8. J. Appl. Crystallogr. 36, 783-786 (2003).

23 Agamalian, M., Wignall, G. D. \& Triolo, R. Optimization of a Bonse-Hart ultra-smallangle neutron scattering facility by elimination of the rocking-curve wings. J. Appl. Crystallogr. 30, 345-352 (1997)

24 Aizawa, K. \& Tomimitsu, H. Design and use of a double-crystal diffractometer for very small-angle neutron-scattering at Jrr-3m. Physica B 213, 884-886 (1995).

25 Schwahn, D., Miksovsky, A., Rauch, H., Seidl, E. \& Zugarek, G. Test of channel-cut perfect crystals for neutron small-angle scattering experiments. Nucl. Instrum. Meth. A 239, 229-234 (1985).

26 Yamaguchi, D., Koizimi, S., Motokawa, R., Kumada, T., Aizawa, K. \& Hashimoto, T. Tandem analyzer crystals systera doubles counting rate for Bonse-Hart ultra-smallangle neutron-scattering spectrometer. Physica B 385-86, 1190-1193 (2006).

27 Choi, S. M., Barker, J. G., Glinka, C. J., Cheng, Y. T. \& Gammel, P. L. Focusing cold neutrons with multiple biconcave lenses for small-angle neutron scattering. J. Appl. Crystallogr. 33, 793-796 (2000)

28 Koizumi, S., Iwase, H., Suzuki, J. I., Oku, T., Motokawa, R., Sasao, H., Tanaka, H. Yamaguchi, D., Shimizu, H. M. \& Hashimoto, T. Focusing and polarized neutron small-angle scattering spectrometer (SANS-J-II). The challenge of observation over length scales from an angstrorm to a micrometre. J. Appl. Crystallogr. 40, S474-S479 (2007).

29 Koizumi, S., Iwase, H., Suzuki, J., Oku, T., Motokawa, R., Sasao, H., Tanaka, H., Yamaguchi, D., Shimizu, H. M. \& Hashimoto, T. Focusing and polarized neutron ultra-small-angle scattering spectrometer (SANS-J-II) at Research Reactor JRR3, Japan. Physica B 385-86, 1000-1006 (2006).

30 Iwase, H., Endo, H., Katagiri, M. \& Shibayama, M. Modernization of the small-angle neutron scattering spectrometer SANS-U by upgrade to a focusing SANS spectrometer. J. Appl. Crystallogr. 44, 558-568 (2011).

31 Alefeld, B., Hayes, C., Mezei, F., Richter, D. \& Springer, T. High-resolution focusing SANS with a toroidal neutron mirror. Physica B 234, 1052-1054 (1997)

32 Brulet, A., Thevenot, V., Lairez, D., Lecommandoux, S., Agut, W., Armes, S. P., Du, J. Z. \& Desert, S. Toward a new lower limit for the minimum scattering vector on the very small angle neutron scattering spectrometer at Laboratoire Leon Brillouin. J. Appl. Crystallogr. 41, 161-166 (2008)

33 Grunzweig, C., Hils, T., Muhlbauer, S., Ay, M., Lorenz, K., Georgii, R., Gahler, R. \& Boni, P. Multiple small angle neutron scattering: a new two-dimensional ultrasmall angle neutron scattering technique. Appl. Phys. Lett. 91 (2007).

34 Beaucage, G. Approximations leading to a unified exponential power-law approach to small-angle scattering. J. Appl. Crystallogr. 28, 717-728 (1995).

35 Beaucage, G. \& Schaefer, D. W. Structural studies of complex-systems using small angle scattering-a unified guinier power-law approach. J. Non-Cryst. Solids 172 797-805 (1994).

36 Guinier, A., Fournet, G., Walker, C. B. F. \& Yudowitch, K. L. Small-Angle Scattering of $X$-Rays (In-house reproduction, 1955).

37 Porod, G. Die rontgenkleinwinkelstreuung von dichtgepackten kolloiden systemen.1. Kolloid Z Z Polym. 124, 83-114 (1951).

38 Porod, G. Die rontgenkleinwinkelstreuung von dichtgepakten kolloiden systemen.2. Kolloid Z Z Polym. 125, 51-57 (1952).

39 Porod, G. Die rontgenkleinwinkelstreuung von dichtgepackten kolloiden systemen.2. Kolloid Z Z Polym. 125, 108-122 (1952).

40 Bale, H. D. \& Schmidt, P. W. Small-angle X-ray-scattering investigation of submicroscopic porosity with fractal properties. Phys. Rev. Lett. 53, 596-599 (1984).

41 Beaucage, G. Small-angle scattering from polymeric mass fractals of arbitrary mass-fractal dimension. J. Appl. Crystallogr. 29, 134-146 (1996).

42 Beaucage, G. Determination of branch fraction and minimum dimension of mass-fractal aggregates. Phys. Rev. E 70 (2004).
43 Pedersen, J. S. Analysis of small-angle scattering data from colloids and polyme solutions: modeling and least-squares fitting. Adv. Colloid. Interfac. 70, 171-210 (1997).

44 Kinning, D. J. \& Thomas, E. L. Hard-sphere interactions between spherical domains in diblock copolymers. Macromolecules 17, 1712-1718 (1984).

45 Wertheim, M. S. Exact solution of Percus-Yevick integral equation for hard spheres. Phys. Rev. Lett. 10, 321-32 (1963).

46 Vrij, A. Mixtures of hard-spheres in the Percus-Yevick approximation-light-scattering at finite angles. J. Chem. Phys. 71, 3267-3270 (1979).

47 Blum, L. \& Stell, G. Polydisperse systems.1. Scattering function for polydisperse fluids of hard or permeable spheres. J. Chem. Phys. 71, $42-46$ (1979).

48 Salacuse, J. J. \& Stell, G. Polydisperse systems-statistical thermodynamics, with applications to several models including hard and permeable spheres. J. Chem. Phys. 77, 3714-3725 (1982)

49 Griffith, W. L., Triolo, R. \& Compere, A. L. Analytical scattering function of a polydisperse Percus-Yevick fluid with schulz-(gamma-) distributed diameters. Phys. Rev. A 35, 2200-2206 (1987)

50 Kotlarchyk, M. \& Chen, S. H. Analysis of small-angle neutron-scattering spectra from polydisperse interacting colloids. J. Chem. Phys. 79, 2461-2469 (1983).

51 Pedersen, J. S. Determination of size distributions from small-angle scattering data for systems with effective hard-sphere interactions. J. Appl. Crystallogr. 27, 595-608 (1994).

52 Hosemann, R. \& Bagchi, S. N. Direct Analysis of Diffraction by Matter (North-Holland Pub., 1962).

53 Debye, P. \& Menke, H. Bestimmung der inneren Struktur von Flüssigkeiten mit Röntgenstrahlen. Physik. Zeitschr 31, 797-798 (1930).

54 Zernike, F. \& Prins, J. A. Die beugung von röntgenstrahlen an flüssingkeiten als effekt der molekülanordnung. Z. Physil. 41, 184-194 (1927).

55 Debye, P. \& Bueche, A. M. Scattering by an inhomogeneous solid. J. Appl. Phys. 20, 518-525 (1949)

56 Gereben, O., Pusztai, L. \& McGreevy, R. L. RMCSANS-modelling the inter-particle term of small angle scattering data via the reverse Monte Carlo method. J. Phys.-Condens. Mat. 22 (2010).

57 Oberdisse, J. Aggregation of colloidal nanoparticles in polymer matrices. Soft Matter $\mathbf{2}$, 29-36 (2006).

58 Oberdisse, J., Hine, P. \& Pyckhout-Hintzen, W. Structure of interacting aggregates of silica nanoparticles in a polymer matrix: small-angle scattering and reverse Monte Carlo simulations. Soft Matter 3, 476-485 (2007).

59 Pusztai, L., Dominguez, H. \& Pizio, O. A. Reverse Monte Carlo modeling of the structure of colloidal aggregates. J. Colloid. Interf. Sci. 277, 327-334 (2004).

60 Toth, G. Monte Carlo determination of the radii and the pair-correlation function of spherical colloids. Physica B 276, 404-405 (2000).

61 Hagita, K., Arai, T., Kishimoto, H., Umesaki, N., Shinohara, Y. \& Amemiya, Y. Two dimensional pattern reverse Monte Carlo method for modelling the structures of nanoparticles in uniaxial elongated rubbers. J. Phys.-Condens. Mat. 19 (2007).

62 Hagita, K., Arai, T., Kishimoto, H., Umesaki, N., Suno, H., Shinohara, Y. \& Amemiya, Y. Structural changes of silica particles in elongated rubber by two-dimensional smallangle X-ray scattering and extended reverse Monte Carlo analysis. Rheol. Acta. 47, 537-541 (2008).

63 Kishimoto, H., Shinohara, Y., Amenya, Y., Inoue, K., Suzuki, Y., Takeuchi, A., Uesugi, K. \& Yagi, N. Structural analysis of filler in rubber composite under stretch with timeresolved two-dimensional ultra-small-angle X-ray scattering. Rubber Chem. Technol. 81, 541-551 (2008).

64 Endo, H., Allgaier, J., Gompper, G., Jakobs, B., Monkenbusch, M., Richter, D. Sottmann, T. \& Strey, R. Membrane decoration by amphiphilic block copolymers in bicontinuous microemulsions. Phys. Rev. Lett. 85, 102-105 (2000).

65 Endo, H., Schwahn, D. \& Colfen, H. On the role of block copolymer additives for calcium carbonate crystallization: small angle neutron scattering investigation by applying contrast variation. J. Chem. Phys. 120, 9410-9423 (2004).

66 Miyazaki, S., Endo, H., Karino, T., Haraguchi, K. \& Shibayama, M. Gelation mechanism of poly(N-isopropylacrylamide)-clay nanocomposite gels. Macromolecules 40, 4287-4295 (2007)

67 Suzuki, T., Endo, H. \& Shibayama, M. Analysis of surface structure and hydrogen/ deuterium exchange of colloidal silica suspension by contrast-variation small-angle neutron scattering. Langmuir 24, 4537-4543 (2008).

68 Takenaka, M., Nishitsuji, S., Amino, N., Ishikawa, Y., Yamaguchi, D. \& Koizumi, S. Structure analyses of swollen rubber-filler systems by using contrast variation SANS. Macromolecules 42, 308-311 (2009).

69 Takenaka, M., Nishitsuji, S., Amino, N., Ishikawa, Y., Yamaguchi, D. \& Koizumi, S Structure analyses of swollen rubber-carbon black systems by using contrast variation small-angle neutron scattering. Rubber Chem. Technol. 85, 157-164 (2012).

70 Koga, T., Hashimoto, T., Takenaka, M., Aizawa, K., Amino, N., Nakamura, M., Yamaguchi, D. \& Koizumi, S. New insight into hierarchical structures of carbon black dispersed in polymer matrices: a combined small-angle scattering study. Macromolecules 41, 453-464 (2008).

71 Koga, T., Takenaka, M., Aizawa, K., Nakamura, M. \& Hashimoto, T. Structure factors of dispersible units of carbon black filler in rubbers. Langmuir 21, 11409-11413 (2005).

72 Fresnais, J., Berret, J. F., Qi, L., Chapel, J. P., Castaing, J. C., Sandre, O., Frka-Petesic, B., Perzynski, R., Oberdisse, J. \& Cousin, F. Universal scattering behavior of coassembled nanoparticle-polymer clusters. Phys. Rev. E 78 (2008).

73 Schaefer, D. W. \& Agamalian, M. M. Ultra-small-angle neutron scattering: a new tool for materials research. Curr. Opin. Solid St. M. 8, 39-47 (2004). 
74 Schaefer, D. W. \& Chen, C. Y. Structure optimization in colloidal reinforcing fillers: precipitated silica. Rubber Chem. Technol. 75, 773-793 (2002).

75 Schaefer, D. W. \& Justice, R. S. How nano are nanocomposites? Macromolecules 40, 8501-8517 (2007)

76 Schaefer, D. W., Rieker, T., Agamalian, M., Lin, J. S., Fischer, D., Sukumaran, S., Chen, C. Y., Beaucage, G., Herd, C. \& Ivie, J. Multilevel structure of reinforcing silica and carbon. J. Appl. Crystallogr. 33, 587-591 (2000).

77 Schaefer, D. W., Suryawanshi, C., Pakdel, P., Ilavsky, J. \& Jemian, P. R. Challenges and opportunities in complex materials: silica-reinforced elastomers. Physica A $\mathbf{3 1 4}$ 686-695 (2002).
78 Ikeda, Y., Higashitani, N., Hijikata, K., Kokubo, Y., Morita, Y., Shibayama, M., Osaka, N., Suzuki, T., Endo, H. \& Kohjiya, S. Vulcanization: new focus on a traditional technology by small-angle neutron scattering. Macromolecules 42, 2741-2748 (2009).

79 Karino, T., Ikeda, Y., Yasuda, Y., Kohjiya, S. \& Shibayama, M. Nonuniformity in natural rubber as revealed by small-angle neutron scattering, small-angle X-ray scattering, and atomic force microscopy. Biomacromolecules 8, 693-699 (2007).

80 Suzuki, T., Osaka, N., Endo, H., Shibayama, M., Ikeda, Y., Asai, H., Higashitani, N., Kokubo, Y. \& Kohjiya, S. Nonuniformity in cross-linked natural rubber as revealed by contrast-variation small-angle neutron scattering. Macromolecules 43, 1556-1563 (2010).

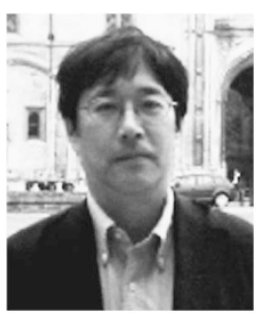

Mikihito Takenaka is currently an Associate Professor of Department of Polymer Chemistry in Kyoto University. He received degrees from the Kyoto University (the master's degree of engineering in 1988 and the doctor's degree of engineering in 1993 with Professor Takeji Hashimoto). Then, he joined the group of Professor Benjamin Chu, the State University of New York at Stony Brook, USA as a postdoctral fellow from 1993 to 1995. From 1995 to 1997, he joined Hashimoto Polymer Phasing project, ERATO, as a postdoctral fellow. In 1997, he was appointed as an Assistant Professor of Department of Polymer Chemistry in Kyoto University, and was promoted to Associate Professor in 2011. His research scope includes the dynamics of phase transitions of polymer alloys, the hierarchical structures of soft matter, the viscoelastic effects of polymeric materials and the directed self-assembling of block copolymer thin films toward nano-patterning. 